Pacific Journal of Mathematics

INTEGRAL EQUATIONS AND PRODUCT INTEGRALS 


\section{INTEGRAL EQUATIONS AND PRODUCT INTEGRALS}

\section{BurRell W. HeltoN}

\section{H. S. Wall, J. S. MacNerney and T. H. Hildebrandt have} shown interdependencies between the equations

$$
f(x)={ }_{a} \Pi^{x}(1+d g) \quad \text { and } \quad f(x)=1+\int_{a}^{x} f d g ;
$$

this paper extends and consolidates some of their results. Let $S$ be a linearly ordered set, $R$ be a normed ring, and $O A^{0}$ and $O M^{0}$ be classes of functions $G$ from $S \times S$ to $R$ for which

$$
\int_{a}^{b}\left|G(I)-\int_{I} G\right|=0
$$

and

$$
\int_{a}^{b}\left|[1+G(I)]-\Pi_{I}(1+G)\right|=0,
$$

respectively. We show the following. If $G$ has bounded variation, $G \in O A^{0}$ if and only if $G \in O M^{0}$. For some rings, the existence of $\int_{a}^{b} G(I)$ and ${ }_{a} \Pi^{b}[1+G(I)]$ imply that $G \in O A^{0}$ and $O M^{0}$, respectively. This is used to prove a product integral solution of integral equations such as

$$
f(x)=f(a)+(R L) \int_{a}^{x}(f G+H f),
$$

where $f$ is a function from $S$ to $R$ and $G$ and $H$ are functions from $S \times S$ to $R$. Then these results are used (a) to show that each nonsingular $m \times m$ matrix of complex numbers has $n$ distinct $n$th roots, (b) to show that, with some restrictions, $\sum_{i=1}^{\infty} A_{i}$ exists if and only if $\prod_{i=1}^{\infty}\left(1+A_{i}\right)$ exists and (c) to find solutions of integrals equations such as

$$
f(x)=f(a)+\int_{a}^{x} f^{n} d g .
$$

In his recent paper, Integral Equations and Semigroups [7], J. S. MacNerney develops some of the interdependencies between additive and multiplicative integration processes for rings, defines two classes $O A$ and $O M$ of functions $V$ and $W$ such that the integral-like formulas

$$
V(a, b)=\int_{a}^{b}(W-1) \quad \text { and } \quad W(a, b)={ }_{a} \Pi^{b}(1+V)
$$

are mutually reciprocal, shows a one-to-one correspondence between the

Received May 8, 1964. 
classes $O A$ and $O M$ and shows that this leads to an integral-equation theory of Cauchy-right and Cauchy-left integrals which extends earlier results of H. S. Wall [9], T. H. Hildebrandt [4] and MacNerney [5] [6]. In several recent papers, W. D. L. Appling [1] [2] has based several proofs on the following theorem: "If $G$ is a real-valued function of subintervals of $[a, b]$ such that $\int_{a}^{b} G(I)$ exists, then

$$
\int_{[a, b]}\left|G(I)-\int_{I} G(J)\right|=0 \text {. }
$$

In this paper we extend this theorem to matrices and to some types of rings, prove a similar theorem for product integrals in which

$$
\int_{[a, b]}\left|[1+G(I)]-\Pi_{I}[1+G(J)]\right|=0
$$

and use these concepts to extend and consolidate some of the theorems of MacNerney [7] and Hildebrandt [4].

We denote by $O A^{0}$ and $O M^{0}$ the classes of functions $G$ satisfying equations 1.1 and 1.2, respectively; MacNerney's class $O A$ is a proper subclass of $O A^{0}$ and $O M$ is closely related to the class $O M^{\circ}$. Using MacNerney's theorems, we prove that, if $G$ has bounded variation, $G \in O A^{0}$ if and only if $G \in O M^{0}$. The defining properties of the classes $O A^{0}$ and $O M^{0}$ are used in the proofs of theorems which give solutions and reciprocal relations for integral equations of the form

$$
f(x)=h(x)+(R L) \int_{a}^{x}[f \cdot H(I)+G(I) \cdot f],
$$

where the range of each function is a subset of a ring $N$. Then these theorems are used

(1) to prove that each nonsingular $m \times m$ matrix of complex numbers has $n$ distinct $n$th roots;

(2) to prove that, with suitable restrictions, $\sum_{i=1}^{\infty} A_{i}$ exists if and only if $\prod_{i=1}^{\infty}\left(1+A_{i}\right)$ exists; and

(3) to find the solutions of integral equations such as

$$
y(x)=y(a)+\int_{a}^{x}\left(y G+y^{n} F\right) .
$$

2. Definitions. Most of the definitions used in this paper are the same as those used by MacNerney [7]; where a new definition is used, the symbol " 0 " is added $\left(O A\right.$ to $\left.O A^{0}\right)$ to indicate a changed, yet similar, definition. Occasionally a phrase or a symbol defined by MacNerney will be used without repeating his definition and will be indicated by $[7, \mathrm{p} . \cdots]$.

0 is a linear ordering $[7$, p. 149] of a nondegenerate set $S . N$ is 
a ring which has a multiplicative identity element denoted by 1 and has a norm $|\cdot|$ with respect to which $N$ is complete and $|1|=1$. Lower case letters will be used for functions from $S$ to a subset of $N$; from $S \times S$ to $N$ by capital letters.

If $G$ is a function from $S \times S$ to $N$ and $\{a, b\} \in S \times S$, the sum (product) integral of $G$ exists on $\{a, b\}$ means there is an element $J$ of $N$ such that if $c$ is a positive number there is an 0 -subdivision $D$ of $\{a, b\}[7, \mathrm{p} .150]$ for which $\left|\sum_{i=1}^{n} G\left(x_{i-1}, x_{i}\right)-J\right|<c\left(\left|\prod_{i=1}^{n} G\left(x_{i-1}, x_{i}\right)-J\right|<c\right)$, provided $\left\{x_{i}\right\}_{i=0}^{n}$ is a refinement of $D\left[7\right.$, p. 150]. $\prod_{i=1}^{n} G\left(x_{i-1}, x_{i}\right)$ means

$$
G\left(x_{0}, x_{1}\right) G\left(x_{1}, x_{2}\right) G\left(x_{2}, x_{3}\right) \cdots G\left(x_{n-1}, x_{n}\right) .
$$

The symbols $\int_{a}^{b} G$ and ${ }_{a} \Pi^{b} G$ will be used to represent the sum and product integrals, respectively.

$O A^{0}$ denotes the set of functions $G$ from $S \times S$ to $N$ such that if $\{a, b\} \in S \times S$ and $\{a, x, y, b\}$ is an 0 -subdivision of $\{a, b\}$ then $\int_{x}^{y} G$ exists and $\int_{a}^{b} H=0$, where $H(x, y)=\left|G(x, y)-\int_{x}^{y} G\right|$.

$O M^{0}$ denotes the set of functions $G$ from $S \times S$ to $N$ such that if $\{a, b\} \in S \times S$ and $\{a, x, y, b\}$ is an 0 -subdivision of $\{a, b\}$, then ${ }_{x} \Pi^{y}(1+G)$ exists and $\int_{a}^{b} K=0$, where $K(x, y)=\left|1+G(x, y)-{ }_{x} \Pi^{y}(1+G)\right|$.

$O B^{0}$ denotes set of functions $G$ from $S \times S$ to $N$ such that if $\{a, b\} \in S \times S$ there is a number $M$ such that, for each 0 -subdivision $\left\{x_{i}\right\}_{i=0}^{n}$ of $\{a, b\}, \sum_{i=1}^{n}\left|G\left(x_{i-1}, x_{i}\right)\right|<M$.

$O P^{0}$ denotes the set of functions $G$ from $S \times S$ to $N$ such that if $\{a, b\} \in S \times S$ there is a number $M$ such that, for each 0 -subdivision $\left\{x_{i}\right\}_{i=0}^{n}$ of $\{a, b\},\left|\prod_{i=p}^{q}\left[1+G\left(x_{i-1}, x_{i}\right)\right]\right|<M$ for $0<p \leqq q \leqq n$. Note that $O B^{0}$ is a proper subset of $O P^{0}$.

The function $G$ from $S \times S$ to $N$ is bounded means if $\{a, b\} \in S \times S$ there is a number $M$ such that if $\{a, x, y, b\}$ is an 0 -subdivision of $\{a, b\}$ then $|G(x, y)|<M$.

Occasionally, statements such as "the function $f(x) G(x, y)$ " will be used as a substitute for the statement "the function $H$ from $S \times S$ to $N$ such that for $\{x, y\} \in S \times S, H(x, y)=f(x) G(x, y)$ ". Similarly, $d f=$ $d f(x, y)=f(y)-f(x)$. Where no misunderstanding is likely, $f_{i}, d f_{i}$ and $G_{i}$ will be used as shortened notations for $f\left(x_{i}\right), f\left(x_{i}\right)-f\left(x_{i-1}\right)$ and $G\left(x_{i-1}, x_{i}\right)$, respectively.

The following special symbols will be used for sum or product integrals of $G$ whenever $G$ is defined in terms of functions $f$ and $H$ from $S$ and $S \times S$, respectively: 


$$
\begin{aligned}
(L) \int_{a}^{b} f H & \sim f(x) H(x, y) ; \\
(R) \int_{a}^{b} f H & \sim f(y) H(x, y) ; \\
2(M) \int_{a}^{b} H f & \sim H(x, y)[f(x)+f(y)] ; \\
(L){ }_{a} \Pi^{b}(1+f H) & \sim 1+f(x) H(x, y) ;
\end{aligned}
$$

etc.

In Theorem 5.8, $\prod_{i=1}^{\infty}\left(1+A_{i}\right)$ exists means $\lim _{n \rightarrow \infty} \prod_{i=1}^{n}\left(1+A_{i}\right)$ and $\left[\lim _{n \rightarrow \infty} \prod_{i=1}^{n}\left(1+A_{i}\right)\right]^{-1}$ exist.

3. $G \in O A^{0}$ only if $G \in O M^{0}$. In this section the properties of functions belonging to $O A^{0}$ and to $O M^{0}$ are studied and some interdependencies of the two sets are shown. The first theorem is an example in which the $O A^{0}$ properties are used; the next two theorems are used in the proofs of later theorems.

Theorem 3.1. If $\{a, b\} \in S \times S$ and $H, F, V, G, K, F_{1}$ and $G_{1}$ are functions from $S \times S$ to $N$ such that

(1) $H, F, G$ and $K$ are bounded,

(2) $F V$ and $V G$ are elements of $O A^{\circ}$, and

(3) for each 0-subdivision $\{a, x, y, b\}$ of $\{a, b\}, F_{1}(x, y)=\int_{x}^{y} F V$ and $G_{1}(x, y)=\int_{x}^{y} V G$,

then $\int_{a}^{b} H F_{1}^{x} G K=\int_{a}^{b} H F V G K=\int_{a}^{b} H F G_{1} K$, provided one of the integrals exist.

Proof. Since $F V$ and $V G$ are elements of $O A^{0}$, then $\int_{a}^{b}\left|F V-F_{1}\right|=0$ and $\int_{a}^{b}\left|V G-G_{1}\right|=0$ and, since $H, F, G$ and $K$ are bounded, it follows that $\int_{a}^{b} H\left(F_{1}-F V\right) G K=0$ and $\int_{a}^{b} H F\left(V G-G_{1}\right) K=0$. Therefore,

$$
\begin{aligned}
\int_{a}^{b} H F_{1} G K & =\int_{a}^{b} H F V G K+\int_{a}^{b} H\left(F_{1}-F V\right) F K \\
& =\int_{a}^{b} H F V G K \\
& =\int_{a}^{b} H F G_{1} K+\int_{a}^{b} H F\left(V G-G_{1}\right) K \\
& =\int_{a}^{b} H F G_{1} K .
\end{aligned}
$$

THEOREM 3.2. If $\{a, b\} \in S \times S$ and $F$ and $G$ are functions from $S \times S$ to $N$ such that $F$ and $G$ are elements of $O P^{0}$, there is a number $M$ such that if $\left\{x_{i}\right\}_{i=0}^{n}$ is an 0-subdivision of $\{a, b\}$, then 


$$
\begin{array}{r}
\left|\prod_{i=1}^{n}\left[1+F\left(x_{i-1}, x_{i}\right)\right]-\prod_{i=1}^{n}\left[1+G\left(x_{i-1}, x_{i}\right)\right]\right| \\
<M^{2} \sum_{i=1}^{n}\left|F\left(x_{i-1}, x_{i}\right)-G\left(x_{i-1}, x_{i}\right)\right|
\end{array}
$$

Proof. Since $F \in O P^{0}$ and $G \in O P^{0}$, there is a number $M$ such that, for each 0 -subdivision $\left\{x_{i}\right\}_{i=0}^{n}$ of $\{a, b\}$ and $1 \leqq p \leqq q \leqq n$,

$$
\left|\prod_{i=p}^{q}\left(1+F_{i}\right)\right|<M \quad \text { and } \quad\left|\prod_{i=p}^{q}\left(1+G_{i}\right)\right|<M \text {. }
$$

From the algebraic identity

$$
\prod_{i=1}^{n} b_{i}-\prod_{i=1}^{n} a_{i}=\sum_{i=1}^{n}\left(\prod_{j=1}^{i-1} a_{j}\right)\left(b_{i}-a_{i}\right)\left(\prod_{t=i+1}^{n} b_{t}\right)
$$

it follows that for each 0 -subdivision $\left\{x_{i}\right\}_{i=0}^{n}$ of $\{a, b\}$

$$
\begin{aligned}
\mid \prod_{i=1}^{n}(1 & \left.+F_{i}\right)-\prod_{i=1}^{n}\left(1+G_{i}\right) \mid \\
& =\left|\sum_{i=1}^{n} \prod_{j=1}^{i-1}\left(1+G_{i}\right)\left(F_{i}-G_{i}\right) \prod_{t=i+1}^{n}\left(1+F_{t}\right)\right| \\
& <\sum_{i=1}^{n} M\left|\left(F_{i}-G_{i}\right)\right| M .
\end{aligned}
$$

THEOREM 3.3. If $\{a, b\} \in S \times S$ and $G$ is a function from $S \times S$ to $N$ such that $G \in O P^{0}, G \in O A^{0}$ and the function $\int_{x}^{y} G \in O P^{0}$, then ${ }_{a} \Pi^{b}(1+G)={ }_{a} \Pi^{b}\left(1+\int G\right)$, provided either product integral exists.

Proof. From Theorem 3.2 there is a number $M$ such that for each 0 -subdivision $\left\{x_{i}\right\}_{i=0}^{n}$ of $\{a, b\}$

$$
\begin{aligned}
\mid \prod_{i=1}^{n}\left(1+\int_{x_{i-1}}^{x_{i}} G\right) & -\prod_{i=1}^{n}\left[1+G\left(x_{i-1}, x_{i}\right)\right] \mid \\
& \leqq M^{2} \sum_{i=1}^{n}\left|\int_{x_{i-1}}^{x_{i}} G-G\left(x_{i-1}, x_{i}\right)\right| .
\end{aligned}
$$

Since $\int_{a}^{b}\left|G-\int G\right|=0$, it follows that ${ }_{a} \Pi^{b}(1+G)={ }_{a} \Pi^{b}\left(1+\int G\right)$, provided one of the product integrals exists.

THEOREM 3.4. If $G$ is a function from $S \times S$ to $N$ such that $G \in O B^{0}$, the following statements are equivalent:

(1) $G \in O A^{0}$ and (2) $G \in O M^{0}$.

Proof. $1 \rightarrow 2$. Let $V$ be the function from $S \times S$ to $N$ such that for each $\{x, y\} \in S \times S, V(x, y)=\int_{x}^{y} G$. By Lemma $3.1[7$, p. 152] there is a function $\alpha$ in $O A^{+}[7, \mathrm{p}$. 150] such that the pair of func- 
tions $\alpha, V$ satisfy the hypothesis of Theorem 3.1 [7, p. 153]; therefore, for each element $\{x, y\}$ of $S \times S,{ }_{x} \Pi^{y}(1+V)$ exists and, by Theorem 3.3 above, ${ }_{x} \Pi^{y}(1+V)={ }_{x} \Pi^{y}(1+G)$. For each 0 -subdivision $\left\{x_{i}\right\}_{i=0}^{n}$ of an element $\{a, b\}$ of $S \times S$

$$
\begin{aligned}
& \sum_{i=1}^{n}\left|{ }_{x_{i-1}} \Pi^{x_{i}}(1+G)-\left[1+G\left(x_{i-1}, x_{i}\right)\right]\right| \\
& \leqq \leqq \sum_{i=1}^{n}\left|{ }_{x_{i-1}} \Pi^{x_{i}}(1+V)-\left[1+V\left(x_{i-1}, x_{i}\right)\right]\right|+\sum_{i=1}^{n}\left|G_{i}-V_{i}\right| \\
& \leqq \sum_{i=1}^{n}||_{x_{i-1}} \Pi^{x_{i}}(1+\alpha)-\left[1+\alpha\left(x_{i-1}, x_{i}\right)\right]\left|+\sum_{i=1}^{n}\right| G_{i}-V_{i} \mid \cdot
\end{aligned}
$$

Since $G \in O A^{0}$ and $\alpha \in O M^{0}$ (by definition and also by our Theorem 4.2), the last member can be made arbitrarily small; therefore

$$
\int_{a}^{b}|(1+G)-\Pi(1+G)|=0
$$

and $G \in O M^{0}$.

$2 \rightarrow 1$. Let $W$ be the function from $S \times S$ to $N$ such that for each element $\{x, y\}$ of $S \times S, W(x, y)={ }_{x} \Pi^{y}(1+G)$. By Lemma 3.2 [7, p. 152] there is a function $\mu \in O M^{+}[7, \mathrm{p} .150]$ such that the pair of functions $\mu, W$ satisfy the hypothesis of Theorem $3.2[7, \mathrm{p}$. 153]; hence, for each element $\{a, b\}$ of $S \times S, \int_{a}^{b}(W-1)$ exists and for each 0 -subdivision $\left\{x_{i}\right\}_{i=0}^{n}$ of $\{a, b\}$,

$$
\begin{aligned}
& \sum_{i=1}^{n}\left|\left[\mu\left(x_{i-1}, x_{i}\right)-1\right]-\int_{x_{i-1}}^{x_{i}}(\mu-1)\right| \\
& \geqq \sum_{i=1}^{n}\left|\left[W\left(x_{i-1}, x_{i}\right)-1\right]-\int_{x_{i-1}}^{x_{i}}(W-1)\right| \\
& =\sum_{i=1}^{n}\left|x_{i-1} \Pi^{x_{i}}(1+G)-1-\int_{x_{i-1}}^{x_{i}}(W-1)\right| \\
& \geqq \sum_{i=1}^{n}\left|G\left(x_{i-1}, x_{i}\right)-\int_{x_{i-1}}^{x_{i}}(W-1)\right| \\
& \quad-\left.\sum_{i=1}^{n}\right|_{x_{i-1}} \Pi^{x_{i}}(1+G)-\left[1+G\left(x_{i-1}, x_{i}\right)\right] \mid .
\end{aligned}
$$

Since $G \in O M^{0}$ and $(\mu-1) \in O A^{0}$ (by definition, also by our Theorem 4.1), it follows that

$$
\begin{gathered}
\int_{a}^{b} G=\int_{a}^{b}(W-1), \\
\int_{a}^{b}\left|G-\int^{b} G\right|=\int_{a}^{b}\left|G-\int(W-1)\right|=0,
\end{gathered}
$$

and $G \in O A^{\circ}$. 
REMARK. $O A^{0}$ is not a subset of $O B^{0}$ and $O B^{0}$ is not a subset of $O A^{0}$. Let $S$ be the set of real numbers. If $h$ is the function from $S$ to $S$ such that $h(x)=1$ for $x$ rational and $h(x)=0$ for $x$ irrational, then the function $H(x, y)=h(y)-h(x)$ is an element of $O A^{0}$ but is not an element of $O B^{0}$. Let $G$ be the function from $S \times S$ to $S$ such that

(1) if $x \leqq y$ then $G(x, y)=y-x$ and

(2) if $x>y$, then $G(x, y)=y-x$ if both $x$ and $y$ are rational, otherwise $G(x, y)=0 . \quad G \in O B^{0}$ and $\int_{0}^{1} G$ exists but $\int_{1}^{0} G$ does not exist and $G \notin O A^{0}$.

MacNerney's Lemma 4.3 [7, p. 156], which is used in the proof of Theorem 3.5, could have been stated as follows:

Lemma $4.3^{\circ}$. If each of $F$ and $G$ is in $O B[7, \mathrm{p} .155]$ and $\alpha$ is a member of $O A^{+}[7$, p. 150] such that $|d F|<\alpha$ and $|d G|<\alpha$ then, for each $\{a, b\}$ in $S \times S,(L) \int_{a}^{b} F d G$ and $(R) \int_{b}^{a} F d G$ exist and-if $e$ is a member of $S$ such that $\{e, a, b\}$ is a subdivision of $\{e, b\}-$

$$
\begin{aligned}
\left|(R) \int_{b}^{a} F d G-F(a) d G(b, a)\right| & =\left|(L) \int_{a}^{b} F d G-F(a) d G(a, b)\right| \\
& \leqq(L) \int_{a}^{b} \alpha(e, \quad) \alpha-\alpha(e, a) \alpha(a, b) .
\end{aligned}
$$

Furthermore, each of the functions

$$
F(x) d G(x, y), \quad F(y) d G(x, y), \quad d G(x, y) F(x)
$$

and $d G(x, y) F(y)$ is an element of $O A^{0}$ and of $O M^{\circ}$.

Indication of proof. MacNerney gives a proof for

$$
\left|(L) \int_{a}^{b} F d G-F(a) d G(a, b)\right| \leqq(L) \int_{a}^{b} \alpha(e, \quad) \alpha-\alpha(e, \alpha) \alpha(a, b) .
$$

The definition of the function $\alpha$ (as well as our Theorem 4.1) assures that the function $\alpha(e, x) \alpha(x, y)$ is an element of $O A^{0}$; therefore $F(x) d G(x, y)$ and $F(y) d G(x, y)$ are elements of $O A^{0}$ and, since the integration by parts theorem (Lemma 4.1, [7, p. 156]) applies, $d G(x, y) F(x)$ and $d G(x, y) F(y)$ are element of $O A^{0}$. It follows from Theorem 3.4 that each of these four functions is an element of $O M^{\circ}$.

THEOREM 3.5. If each of $f_{1}, f_{2}, f_{3}$ and $f_{4}$ is a function from $S$ to $N$ and $d f_{i} \in O B^{0}$ for $i=1,2,3,4$, the function $G$ is an element of $O B^{0}$ and $O A^{0}$ and $H$ is the function

$$
H(x, y)=\left[f_{1}(x)+f_{2}(y)\right] G(x, y)\left[f_{3}(x)+f_{4}(y)\right],
$$


then $H \in O A^{0}$ and $H \in O M^{0}$.

Proof. Let $V$ be the function $V(x, y)=\int_{x}^{y} G$. From Lemma $4.3^{\circ}$ it follows that each of the functions $f_{1}(x) V(x, y), f_{2}(y) V(x, y), V(x, y) f_{3}(x)$ and $V(x, y) f_{4}(y)$ is an element of $O A^{0}$. Since $f_{1}(x) V(x, y)$ and $G$ are elements of $O A^{0}$, and

$$
\begin{aligned}
& \sum_{D}\left|\int_{x_{i-1}}^{x_{i}} f_{1}() V(, \quad)-f_{1}(x) V(x, y)\right| \\
& \quad=\sum_{D}\left|\int_{x_{i-1}}^{x_{i}} f_{1}() V(, \quad)-f_{1}(x) G(x, y)+f_{1}(x) G(x, y)-f_{1}(x) V(x, y)\right| \\
& \quad \geqq \sum_{D}\left|\int_{x_{i-1}}^{x_{i}} f_{1}() V(, \quad)-f_{1}(x) G(x, y)\right|-\sum_{D}\left|f_{1}(x)\right||G(x, y)-V(x, y)|
\end{aligned}
$$

it follows that $f_{1}(x) G(x, y) \in O A^{0}$. Similarly, the other three composite functions of $f_{i}$ and $G$ are elements of $O A^{0}$. $H \in O A^{0}$ because $O A^{0}$ is closed with respect to addition. Since $H \in O B^{0}$, it follows from Theorem 3.4 that $H \in O M^{0}$.

REMARK. If $H$ is the function

$$
H(x, y)=\sum_{i=1}^{n} F_{i}(x, y) G_{i}(x, y) H_{i}(x, y)
$$

where, for $i=1,2,3, \cdots, n, G_{i}$ is an element of $O B^{0}$ and $O A^{0}$ and each of the functions $F_{i}$ and $H_{i}$ is composed of products and sums of functions $f$ from $S$ to $N$ for which $d f \in O B^{0}$, then, by using Theorems 3.1 and 3.5 , it follows that $H \in O A^{0}$ and $H \in O M^{0}$.

4. $O A^{0}, O M^{0}$ and a special ring. In this section it is shown that if suitable restrictions are placed on the ring $N$, then the existence of the sum integral $\int_{a}^{b} G$ implies that $G \in O A^{0}$ and the existence of the product integral $\left.\Pi_{a} \Pi^{(1}+G\right)$ implies that $G \in O M^{0}$.

Let $R$ be a ring which has $K$ subsets $R_{1}, R_{2}, \cdots, R_{K}$ such that $R=\bigcup_{i=1}^{K} R_{i}$ and $R$ has a norm $|\cdot|$ with respect to which $R$ is complete and $|1|=1$ and, if $A$ and $B$ are elements of the same subset $R_{i}$ of $R$, then $|A+B|=|A|+|B|$. The field of complex numbers with the norm of $(a, b)$ defined as $|a|+|b|$ satisfies these conditions.

THEOREM 4.1. If $G$ is a function from $S \times S$ to $R$ such that $\int_{x}^{y} G$ exists for each element $\{x, y\}$ of $S \times S$, then $G \in O A^{0}$.

Proof. If the theorem is false and $H(x, y)=\int_{x}^{y} G-G(x, y)$ for each element $\{x, y\}$ of $S \times S$, then there is an element $\{a, b\}$ of $S \times S$, 
a positive number $c$ and an 0 -subdivision $D=\left\{x_{i}\right\}_{i=0}^{n}$ of $\{a, b\}$ such that $\sum_{i=1}^{n}\left|H\left(x_{i-1}, x_{i}\right)\right|>c$ and if $\left\{y_{j}\right\}_{j=1}^{p}$ is a refinement of $D$, then

$$
\left|\int_{a}^{b} G-\sum_{j=1}^{p} G\left(y_{j_{-1}}, y_{j}\right)\right|<c / 2 K \text {. }
$$

The following definitions are used to define a refinement $D^{\prime}$ of $D$ which will lead to a contradiction. For each integer $k, 1 \leqq k \leqq K$, let $A_{k}$ be the set of integers such that $p \in A_{k}$ only if $H\left(x_{p_{-1}}, x_{p}\right) \in R_{k}$. Since $\sum_{i=1}^{n}\left|H\left(x_{i-1}, x_{i}\right)\right|>c$, there is an element $A$ of $\left\{A_{k}\right\}_{k=1}^{R}$ such that $\sum_{i \in A}\left|H\left(x_{i-1}, x_{i}\right)\right|>c / K$. For each integer $i, 1 \leqq i \leqq n$, let $D_{i}$ be a subdivision of $\left\{x_{i-1}, x_{i}\right\}$ and $k_{i}$ be an element of $R$ such that

(1) if $i \in A$, then $D_{i}=\left\{x_{i-1}, x_{i}\right\}$ and $k_{i}=H\left(x_{i-1}, x_{i}\right)$; and

(2) if $i \notin A$, then $D_{i}$ is an 0 -subdivision of $\left\{x_{i-1}, x_{i}\right\}$ such that $k_{i}=\int_{x_{i-1}}^{x_{i}} G-\sum_{D_{i}} G\left(x_{j-1}, x_{j}\right)$ and $\left|k_{i}\right|<c /\left(2^{i} \cdot 2 K\right)$. Let $D^{\prime}=\bigcup_{i=1}^{K} D_{i}$; then $D^{\prime}$ is a refinement of $D$ and

$$
\begin{aligned}
c / 2 K>\left|\int_{a}^{b} G-\sum_{D^{\prime}} G\left(x_{j_{-1}}, x_{j}\right)\right| & =\left|\sum_{D} \int_{x_{i-1}}^{x_{i}} G-\sum_{D}\left(\sum_{D_{i}} G_{j}\right)\right| \\
& =\left|\sum_{D}\left(\int_{x_{i-1}}^{x_{i}} G-\sum_{D_{i}} G_{j}\right)\right| \\
& =\left|\sum_{D} k_{i}\right| \geqq\left|\sum_{i \in A} k_{i}\right|-\sum_{i \notin A}\left|k_{i}\right| \\
& >\left|\sum_{i \in A} k_{i}\right|-\sum_{i \notin A} c /\left(2^{i} \cdot 2 K\right) ;
\end{aligned}
$$

hence $c / K>\left|\sum_{i \in A} H\left(x_{i-1}, x_{i}\right)\right|=\sum_{i \in A}\left|H_{i}\right|>c / K$. This contradiction proves that $\int_{a}^{b}|H|=0$ and that $G \in O A^{0}$.

THEOREM 4.2. If $G$ is a function from $S \times S$ to $R$ such that for $\{a, b\} \in S \times S$ there is a number $M$ such that, for each 0 -subdivision $\{a, x, y, b\}$ of $\{a, b\},{ }_{x} \Pi^{y}(1+G)$ and $\left[{ }_{x} \Pi^{y}(1+G)\right]^{-1}$ exist and are bounded by $M-1$, then $G \in O M^{0}$.

Proof. For each element $\{x, y\}$ of $S \times S$ let $F(x, y)=1+G(x, y)$ and $H(x, y)={ }_{x} \Pi^{y}(1+G)-[1+G(x, y)]$. If the theorem is false, there is an element $\{a, b\} \in S \times S$, a positive number $c$ and an 0 -subdivision $D=\left\{x_{i}\right\}_{i=0}^{n}$ of $\{a, b\}$ such that $\sum_{i=1}^{n}\left|H\left(x_{i-1}, x_{i}\right)\right|>c$ and, if $\left\{y_{j}\right\}_{j=1}^{p}$ is a refinement of $D$ and $1 \leqq i \leqq n$, then

$$
\begin{gathered}
\left|{ }_{a} \Pi^{b} F-\prod_{j=1}^{p} F\left(y_{j-1}, y_{j}\right)\right|<c /\left(4 M^{2} K\right) \\
\left|\prod_{j=q_{i}}^{p} F\left(y_{j_{-1}}, y_{j}\right)\right|<M,
\end{gathered}
$$




$$
\left|{ }_{x_{i}} \Pi^{b} F-\Pi_{j=q_{i}}^{p} F\left(y_{j_{-1}}, y_{j}\right)\right|<1 /\left(2 M^{3}\right),
$$

where $y_{j-1}=x_{i}$ for $j=q_{i}$.

The following definitions are used to define a refinement $D^{\prime}$ of $D$ which will lead to a contradiction. For each integer $k, 1 \leqq k \leqq K$, let $A_{k}$ be the set of integers such that $i \in A_{k}$ only if

$$
\left({ }_{a} \Pi^{x_{i-1}} F\right) H\left(x_{i-1}, x_{i}\right)\left(x_{i+1} \Pi^{b} F\right)
$$

is an element of $R_{k}$. Since $\sum_{i=1}^{n}\left|H\left(x_{i-1}, x_{i}\right)\right|>c$, there is an element $A$ of $\left\{A_{k}\right\}_{k=1}^{K}$ such that $\sum_{i \in A}\left|H\left(x_{i-1}, x_{i}\right)\right|>c / K$. For each integer $i$, $1 \leqq i \leqq n$, let $D_{i}$ be a subdivision of $\left\{x_{i-1}, x_{i}\right\}$ and $k_{i}$ be an element of $R$ such that

(1) if $i \in A$, then $D_{i}=\left\{x_{i-1}, x_{i}\right\}$ and $k_{i}=H\left(x_{i-1}, x_{i}\right)$; and

(2) if $i \notin A$, then $D_{i}$ is an 0 -subdivision $\left\{z_{j}\right\}_{j=0}^{p_{i}}$ of $\left\{x_{i-1}, x_{i}\right\}$ such that $k_{i}={ }_{x_{i-1}} \Pi{ }^{x_{i}} F-\prod_{j=1}^{p_{i}} F\left(z_{j_{-1}}, z_{j}\right)$ and $\left|k_{i}\right|<c /\left(2^{i} 4 M^{4} K\right)$.

Let $D^{\prime}=\bigcup_{i=1}^{n} D_{i}=\left\{y_{j}\right\}_{j=1}^{p}$; then $D^{\prime}$ is a refinement of $D$ and

$$
\begin{aligned}
c /\left(4 M^{2} K\right) & >\left|\prod_{j=1}^{p} F\left(y_{j-1}, y_{j}\right)-{ }_{a} \Pi^{b} F\right| \\
& =\left|\prod_{D}\left[\prod_{D_{i}} F\left(y_{j-1}, y_{j}\right)\right]-\prod_{\nu}\left(x_{i-1} \Pi^{x_{i}} F\right)\right| \\
& =\left|\sum_{D}^{n}{ }_{i=1}\left(\Pi_{a} \Pi^{x_{i-1}} F\right) k_{i} \prod_{j=i+1}^{n} \Pi_{D_{j}} F\left(y_{k-1}, y_{k}\right)\right| \\
& \geqq\left|\sum_{i \in A}[\cdot]\right|-\sum_{i \notin A}\left|{ }_{a} \Pi^{x_{i-1}} F\right| \cdot\left|k_{i}\right| \cdot\left|\prod_{j=i+1}^{n} \prod_{D_{j}} F_{k}\right| \\
& >\left|\sum_{i \in A}[\cdot]\right|-M \sum_{i \notin A}\left|k_{i}\right| M
\end{aligned}
$$

hence

$$
\begin{aligned}
\sum_{i \in A}\left|k_{i}\right| /\left(2 M^{2}\right)> & c /\left(2 M^{2} K\right) \\
> & \left|\sum_{i \in A}\left({ }_{a} \Pi^{x_{i-1}} F\right) k_{i} \prod_{j=i+1}^{n} \prod_{D_{j}} F_{k}\right| \\
\geqq & \left|\sum_{i \in A} \Pi^{x_{i-1}} F k_{i x_{i+1}} \Pi^{b} F\right| \\
& -\sum_{i \in A}\left|{ }_{a} \Pi^{x_{i-1}} F\right| \cdot\left|k_{i}\right| \cdot\left|\prod_{j=i+1}^{n} \prod_{D_{j}} F_{k}-{ }_{x_{i+1}} \Pi^{b} F\right| \\
> & \left|\sum_{i \in A}\left(\Pi_{a} \Pi^{x_{i-1}} F\right) k_{i}\left(x_{i+1} \Pi^{b} F\right)\right| \\
& -M \sum_{i \in A}\left|k_{i}\right|\left(1 / 2 M^{3}\right) .
\end{aligned}
$$

Therefore 


$$
\begin{aligned}
\left(1 / M^{2}\right) \sum_{i \in A}\left|k_{i}\right| & >\left|\sum_{i \in A}\left({ }_{a} \Pi^{x_{i-1}} F\right) k_{i}\left(x_{i+1} \Pi^{b} F\right)\right| \\
& =\sum_{i \in A}\left|\left({ }_{a} \Pi^{x_{i-1}} F\right) k_{i}\left(x_{i+1} \Pi^{b} F\right)\right|
\end{aligned}
$$

(See def. of $A_{k}, A$ and $k_{i}$.)

$$
\begin{aligned}
& \left.\geqq\left(1 / M^{2}\right) \sum_{i \in A} \mid{ }_{a} \Pi^{x_{i-1}} F\right)^{-1} \mid \\
& \quad \quad \times\left|\left({ }_{a} \Pi^{x_{i-1}} F\right) k_{i}\left(x_{x_{i+1}} \Pi^{b} F\right)\right| \cdot\left|\left(_{x_{i+1}} \Pi^{b} F\right)^{-1}\right| \\
& \geqq\left(1 / M^{2}\right) \sum_{i \in A}\left|k_{i}\right| \cdot
\end{aligned}
$$

This contradiction proves that $\int_{a}^{b}|H|=0$ and that $G \in O M^{0}$.

5. Integral equations; special uses. In this section several theorems are proved showing that product integrals can be used in solving integral equations. Then these theorems are used to show that a nonsingular $m \times m$ matrix of complex numbers has $n$ distinct $n$th roots and that, with some restrictions, $\sum_{i=1}^{\infty} A_{i}$ exists if and only if $\prod_{i=1}^{\infty}\left(1+A_{i}\right)$ exists. The first four theorems show interdependencies between equations containing sum integrals and equations containing product integrals. Since the proofs of these theorems are similar except for minor algebraic manipulations, the proof for Theorem 5.1 is given and the other proofs are omitted. We are indebted to the referee for the proof of the following lemma which is used in the proofs of the next four theorems.

Lemma. Suppose the functions $F, G$ and $H$ are elements of $O B^{0}$ such that $F \in O A^{0}$ and ${ }_{x} \Pi^{y}(1+H),{ }_{y} \Pi^{x}(1+G)$ and

$$
(R R) \int_{x}^{y} \Pi^{t}(1+G) \cdot F \cdot{ }_{t} \Pi^{y}(1+H)
$$

exist for $\{x, y\} \in S \times S$. If $\{a, b\} \in S \times S$, then

$$
\int_{a}^{b}\left|F(x, y)-(R R) \int_{x}^{y} \Pi^{t}(1+G) \cdot F \cdot{ }_{t} \Pi^{y}(1+H)\right|=0 \text {. }
$$

Proof. Let $\{a, b\}$ be in $S \times S, M$ be a positive number, and $V$ and $W$ be functions such that if $\{a, x, y, b\}$ is an 0 -subdivision of $\{a, b\}$ then $\left|{ }_{y} \Pi^{x}(1+G)\right|<M,\left.\right|_{x} \Pi^{y}(1+H) \mid<M$,

$$
V(x, y)=\int_{x}^{y} F
$$

and

$$
W(x, y)=(R R) \int_{x}^{y} \Pi^{t}(1+G) \cdot F \cdot{ }_{t} \Pi^{y}(1+H) .
$$


Since $F$ is in $O A^{0}$ it is clear that if $\{a, x, y, b\}$ is an 0 -subdivision of $\{a, b\}$ then

$$
W(x, y)=(R R) \int_{x}^{y} \Pi^{t}(1+G) \cdot V \cdot{ }_{t} \Pi^{y}(1+H),
$$

and that the assertion of the lemma is equivalent to this:

$$
\int_{a}^{b}|V-W|=0
$$

Since $H$ is in $O B^{0}$, there exists a function $\beta$ such that if $\{a, x, y, b\}$ is an 0 -subdivision of $\{a, b\}$ then $\beta(x, y)$ is the least number $Q$ such that, for each 0-subdivision $\left\{t_{p}\right\}_{0}^{n}$ of $\{x, y\}, \sum_{1}^{n}\left|H\left(t_{p_{-1}}, t_{p}\right)\right| \leqq Q$. If $\{a, x, y, z, b\}$ is an 0 -subdivision of $\{a, b\}$ then $\beta(x, y)+\beta(y, z) \leqq \beta(x, z)$; hence noting that if $\left\{t_{p}\right\}_{0}^{n}$ is an 0 -subdivision of $\{x, y\}$ then

$$
\sum_{1}^{n}\left|H\left(t_{p_{-1}}, t_{p}\right)\right| \leqq \sum_{1}^{n} \beta\left(t_{p_{-1}}, t_{p}\right),
$$

one readily finds that $\left|1-{ }_{x} \Pi^{y}(1+H)\right| \leqq M \int_{x}^{y} \beta$. Similarly there is a function $\alpha$ such that if $\{a, x, y, b\}$ is an 0 -subdivision of $\{a, b\}$ then $\alpha(x, y)$ is the least number $Q$ such that, for each 0 -subdivision $\left\{t_{p}\right\}_{0}^{n}$ of $\{x, y\}, \sum_{1}^{n}\left|G\left(t_{p}, t_{p_{-1}}\right)\right| \leqq Q$, and $\left|1-{ }_{y} \Pi^{x}(1+G)\right| \leqq M \int_{x}^{y} \alpha$.

Suppose that $c>0$; let

$$
d\left\{1+M \int_{a}^{b} \beta+M^{2} \int_{a}^{b} \alpha+\left(M+M^{2}\right) \int_{a}^{b}|V|\right\}=c,
$$

and $n$ be a positive integer such that

$$
\int_{a}^{b} \alpha<n d, \quad \int_{a}^{b} \beta<n d, \quad \text { and } \quad \int_{a}^{b}|V|<n d .
$$

Now, let $[a, b]$ denote the subset of $S$ to which $u$ belongs only in case $\{a, u, b\}$ is an 0 -subdivision of $\{a, b\}$, and let $A$ be the collection to which $X$ belongs only in case either

(1) there is a positive integer $i$ less than $n$ such that $X$ is the subset of $[a, b]$ to which $u$ belongs only in case

$$
(i-1) \int_{a}^{b} \alpha \leqq(n) \int_{a}^{u} \alpha<(i) \int_{a}^{b} \alpha,
$$

or (2) $X$ is the subset of $[a, b]$ to which $u$ belongs only in case

$$
(n-1) \int_{a}^{b} \alpha \leqq(n) \int_{a}^{u} \alpha \leqq(n) \int_{a}^{b} \alpha .
$$


Note that $A$ is a finite collection of mutually exclusive subsets of $[a, b]$ filling up $[a, b]$ such that

(1) if $X$ and $Y$ are members of $A$, then either $\{u, v\}$ is in 0 for each $u$ in $X$ and each $v$ in $Y$, or $\{v, u\}$ is in 0 for each $u$ in $X$ and each $v$ in $Y$, and

(2) if $\{a, u, v, b\}$ is an 0 -subdivision of $\{a, b\}$ and there is a member of $A$ to which both $u$ and $v$ belong then $\int_{u}^{v} \alpha<d$.

Let $B$ be the collection obtained in this way by considering $\int_{a}^{u} \beta$, and $C$ the corresponding collection obtained by considering $\int_{a}^{a}|V| \cdot{ }^{a}$ Let $D$ be the collection to which $T$ belongs only in case there exists a member $X$ of $A$, a member $Y$ of $B$, and a member $Z$ of $C$ such that $T$ is the set to which $u$ belongs only in case $u$ belongs to $X$, to $Y$, and to $Z$. Now, $D$ is a finite collection of mutually exclusive subsets of $[a, b]$ filling up $[a, b]$ such that

(1) if $X$ and $Y$ are members of $D$, then either $\{u, v\}$ is in 0 for each $u$ in $X$ and each $v$ in $Y$, or $\{v, u\}$ is in 0 for each $u$ in $X$ and $v$ in $Y$, and

(2) if $\{a, u, v, b\}$ is an 0 -subdivision of $\{a, b\}$ and there is a member of $D$ to which both $u$ and $v$ belong then

$$
\int_{u}^{v} \alpha<d, \quad \int_{u}^{v} \beta<d, \quad \text { and } \quad \int_{u}^{v}|V|<d
$$

If $D$ has only one member, let $t$ be the 0 -subdivision $\{a, b\}$ of $\{a, b\}$. Otherwise, let $N$ be an integer such that $D$ has only $N+1$ members, $\left\{X_{p}\right\}_{0}^{N}$ be a simple ordering of $D$ such that if $u_{p}$ is in $X_{p}$ $(p=0, \cdots, N)$ then $\left\{a, u_{0}, \cdots, u_{N}, b\right\}$ is an 0 -subdivision of $\{a, b\}$, and $\left\{t_{p}\right\}_{0}^{N}$ be an 0 -subdivision of $\{a, b\}$ such that $t_{p}$ is in $X_{p}(p=0, \cdots, N)$. Let $\left\{r_{p}\right\}_{0}^{m}$ be a refinement of $t$.

Suppose, temporarily, that $p$ is a positive integer less than $m+1$, and let $\left\{s_{i}\right\}_{0}^{k}$ be an 0 -subdivision of $\left\{r_{p_{-1}}, r_{p}\right\}$ such that

$$
\left|W\left(r_{p-1}, r_{p}\right)-\sum_{1}^{k}{ }_{r_{p}} \Pi^{s_{i}}(1+G) \cdot V\left(s_{i-1}, s_{i}\right) \cdot{ }_{s_{i}} \Pi^{r_{p}}(1+H)\right|<d / m .
$$

Denote

$$
V\left(r_{p-1}, r_{p}\right)-\sum_{1}^{k} r_{p} \Pi^{s_{i}}(1+G) \cdot V\left(s_{i-1}, s_{i}\right) \cdot{ }_{s_{i}} \Pi^{r_{p}}(1+H)
$$

by $U$, so that

$$
\begin{aligned}
|U| & =\left|\sum_{1}^{k}\left\{V\left(s_{i-1}, s_{i}\right)-{ }_{r_{p}} \Pi^{s_{i}}(1+G) \cdot V\left(s_{i-1}, s_{i}\right) \cdot s_{i} \Pi^{r_{p}}(1+H)\right\}\right| \\
& \leqq \sum_{1}^{k}\left|V\left(s_{i-1}, s_{i}\right)\right|
\end{aligned}
$$




$$
\begin{aligned}
& \times\left\{\left|1-{ }_{s_{i}} \Pi^{r_{p}}(1+H)\right|+\left.\left|1-{ }_{r_{n}} \Pi^{s_{i}}(1+G)\right|\right|_{s_{i}} \Pi^{r_{p}}(1+H) \mid\right\} \\
\leqq & \sum_{1}^{k}\left|V\left(s_{i-1}, s_{i}\right)\right|\left\{M \int_{s_{i}}^{r_{p}} \beta+M^{2} \int_{s_{i}}^{r_{p}} \alpha\right\} .
\end{aligned}
$$

If there exists a member of $D$ to which each of $r_{p_{-1}}$ and $r_{p}$ belongs, then

$$
\begin{aligned}
|U| & \leqq \sum_{1}^{k}\left|V\left(s_{i-1}, s_{i}\right)\right|\left\{M \int_{r_{p-1}}^{r_{p}} \beta+M^{2} \int_{r_{p-1}}^{r_{p}} \alpha\right\} \\
& \leqq d\left\{M \int_{r_{p-1}}^{r_{p}} \beta+M^{2} \int_{r_{p-1}}^{r_{p}} \alpha\right\} .
\end{aligned}
$$

Otherwise, there exist members $X$ and $Y$ of $D$ and a positive integer $j$ in $[0, m]$ such that $s_{i}$ is in $X$ for $0 \leqq i<j$ and $s_{i}$ is in $Y$ for $j \leqq i \leqq m$, whence

$$
\begin{aligned}
|U| \leqq & \sum_{1}^{j-1}\left|V\left(s_{i-1}, s_{i}\right)\right|\left\{M \int_{r_{p-1}}^{r_{p}} \beta+M^{2} \int_{r_{p-1}}^{r_{p}} \alpha\right\} \\
& +\sum_{j}^{m}\left|V\left(s_{i-1}, s_{i}\right)\right|\left\{M \int_{s_{j}}^{r_{p}} \beta+M^{2} \int_{s_{j}}^{r_{p}} \alpha\right\} \\
\leqq & d\left\{M \int_{r_{p-1}}^{r_{p}} \beta+M^{2} \int_{r_{p-1}}^{r_{p}} \alpha\right\}+\int_{r_{p-1}}^{r_{p}}|V|\left\{M d+M^{2} d\right\} .
\end{aligned}
$$

Therefore, in either case,

$$
\begin{aligned}
& \left|V\left(r_{p_{-1}}, r_{p}\right)-W\left(r_{p_{-1}}, r_{p}\right)\right| \\
& \quad<d\left\{1 / m+M \int_{r_{p-1}}^{r_{p}} \beta+M^{2} \int_{r_{p-1}}^{r_{p}} \alpha+\left(M+M^{2}\right) \int_{r_{p-1}}^{r_{p}}|V|\right\} .
\end{aligned}
$$

From the considerations of the preceding paragraph it follows that

$$
\begin{aligned}
& \sum_{1}^{m}\left|V\left(r_{p_{-1}}, r_{p}\right)-W\left(r_{p-1}, r_{p}\right)\right| \\
& \quad<d\left\{1+M \int_{a}^{b} \beta+M^{2} \int_{a}^{b} \alpha+\left(M+M^{2}\right) \int_{a}^{b}|V|\right\}=c,
\end{aligned}
$$

and the proof is complete.

\section{TheOREM 5.1. Suppose}

(1) $a \in S$, $f$ and $h$ are functions from $S$ to $N$ such that $f(a)=$ $h(a)$ and $d h \in O B^{0}$,

(2) $G, H, A$ and $B$ are functions from $S \times S$ to $N$ such that $(1-H)^{-1}$ exists and is $B, B$ is bounded, $(d h) B \in O A^{0}$, and for $\{x, y\} \in S \times S$ $A(x, y)=[1+G(x, y)][1-H(x, y)]^{-1}$ and $A-1 \in O B^{0}$, and

(3) $h$ is a constant function or ${ }_{x} \Pi^{y} A$ exists for each $\{x, y\} \in S \times S$. The following statements are equivalent:

(1) $f(y) H(x, y)+f(x) G(x, y) \in O A^{0}$ and 


$$
f(x)=h(x)+(R L) \int_{a}^{x}(f H+f G)
$$

for each element $\{a, x\}$ of $S \times S$; and

(2) if $\{a, x, y\}$ is an 0 -subdivision of $\{a, y\} \in S \times S$, then

$$
(L) \int_{a}^{y}|f()[\Pi A-A(,)]|=0
$$

and

$$
f(y)=f(x){ }_{x} \Pi^{y} A+(R) \int_{x}^{y}\left[(d h) B_{t} \Pi^{y} A\right] .
$$

Furthermore, if $f^{-1}$ exists and is bounded, then $(A-1) \in O M^{0}$.

Proof. We will consider the statements concerning $O A^{0}$ and $O M^{0}$ after the other parts of the theorem have been proved.

$1 \rightarrow 2$. If $\{a, y\} \in S \times S,\{a, x, y\}$ and $\left\{x_{i}\right\}_{i=0}^{n}$ are 0 -subdivisions of $\{a, y\}$ and of $\{x, y\}$, respectively, and $1 \leqq i \leqq n$, then

$$
f\left(x_{i}\right)-f\left(x_{i-1}\right)=h\left(x_{i}\right)-h\left(x_{i-1}\right)+(R L) \int_{x_{i-1}}^{x_{i}}(f H+f G) ;
$$

hence

$$
f_{i}=f_{i-1}+d h_{i}+f_{i} H_{i}+f_{i-1} G_{i}+c_{i}
$$

where $d h_{i}=h\left(x_{i}\right)-h\left(x_{i-1}\right)$ and

$$
c_{i}=(R L) \int_{x_{i-1}}^{x_{i}}(f H+f G)-\left(f_{i} H_{i}+f_{i-1} G_{i}\right) ;
$$

hence,

$$
\begin{aligned}
f_{i} & =d h_{i}\left(1-H_{i}\right)^{-1}+f_{i-1}\left(1+G_{i}\right)\left(1-H_{i}\right)^{-1}+c_{i}\left(1-H_{i}\right)^{-1} \\
& =f_{i-1} A_{i}+\left(d h_{i}+c_{i}\right) B_{i} .
\end{aligned}
$$

By iteration, using $i=1,2, \cdots, n$ in order, we obtain

$$
f_{n}=f_{0} \prod_{i=1}^{n} A_{i}+\sum_{i=1}^{n} d h_{i} B_{i} \prod_{j=i+1}^{n} A_{j}+\sum_{i=1}^{m} c_{i} B_{i} \prod_{j=i+1}^{n} A_{j} \cdot
$$

Since the product integral ${ }_{x} \Pi^{y} A$ exists, $d h \in O B^{0}, B$ is bounded, $(A-1) \in O P^{0}$ and $\sum_{i=1}^{n}\left|c_{i}\right|$ can be made arbitrarily small, it follows that $(R) \int_{x}^{y}(d h) B_{x} \Pi^{y} A$ exists and that

$$
f(y)=f(x)_{x} \Pi^{y} A+(R) \int_{x}^{y}(d h) B_{t} \Pi^{y} A .
$$

Equation 5.12 shows that if $h$ is a constant function, the requirement that ${ }_{x} \Pi^{y} A$ exists is not needed. 
$2 \rightarrow 1$. If $\{a, x\} \in S \times S,\left\{x_{i}\right\}_{i=0}^{n}$ is an 0 -subdivision of $\{a, x\}$ and $1 \leqq i \leqq n$, then

$$
\begin{aligned}
f\left(x_{i}\right) & =f\left(x_{i-1}\right)_{x_{i-1}} \Pi^{x_{i}} A+(R) \int_{x_{i-1}}^{x_{i}}(d h) B_{t} \Pi^{x_{i}} A \\
& =f_{i-1}\left(1+G_{i}\right)\left(1-H_{i}\right)^{-1}+f_{i-1} d_{i}+d h_{i} B_{i}+e_{i},
\end{aligned}
$$

where

$$
d_{i}={ }_{x_{i-1}} \Pi^{x_{i}} A-A\left(x_{i-1}, x_{i}\right)
$$

and

$$
e_{i}=\int_{x_{i-1}}^{x_{i}}\left[(d h) B_{t} \Pi^{x_{i}} A\right]-d h_{i} B_{i}
$$

Hence,

$$
\begin{aligned}
f_{i}\left(1-H_{i}\right)= & f_{i-1}\left(1+G_{i}\right) \\
& +f_{i-1} d_{i}\left(1-H_{i}\right)+d h_{i}+e_{i}\left(1-H_{i}\right),
\end{aligned}
$$

and

$$
\begin{aligned}
f_{i}-f_{i-1}= & f_{i} H_{i}+f_{i-1} G_{i} \\
& +f_{i-1} d_{i}\left(1-H_{i}\right)+d h_{i}+e_{i}\left(1-H_{i}\right) .
\end{aligned}
$$

Since $\sum_{i=1}^{n}\left|f_{i-1} d_{i}\right|$ and, by using the lemma, $\sum_{i=1}^{n}\left|e_{i}\right|$ can be made arbitrarily small, it follows that

$$
f(x)=h(x)+(R L) \int_{a}^{x}(f H+f G) .
$$

To prove the statements concerning the sets $O M^{0}$ and $O A^{0}$, we combine equations 5.11 and 5.13 and obtain

$$
c_{i}=f_{i-1} d_{i}\left(1-H_{i}\right)+e_{i}\left(1-H_{i}\right) \text {. }
$$

A review of the definitions of $c_{i}, d_{i}$ and $e_{i}$ shows that if $c$ and $d$ are defined as functions, then $\int_{a}^{x}|c|=0$ if and only if $(L) \int_{a}^{x}|f d|=0$; also, $\int_{a}^{x}|d|=0$ if $f^{-1}$ exists and is bounded.

REMARK. If $N$ is the field of complex numbers, then

$$
\prod_{D} A_{i}=\prod_{D}\left(1+G_{i}\right)\left(1-H_{i}\right)^{-1}=\prod_{D}\left(1+G_{i}\right) / \prod_{D}\left(1-H_{i}\right)
$$

and

$$
{ }_{a} \Pi^{x} A={ }_{a} \Pi^{x}(1+G) /{ }_{a} \Pi^{x}(1-H) ;
$$

for the special case where 


$$
\begin{gathered}
(R L) \int_{a}^{x}(f H+f G)=(M) \int_{a}^{x} f F \\
{ }_{a} \Pi^{x} A={ }_{a} \Pi^{x}\left(1+\frac{1}{2} F\right) /{ }_{a} \Pi^{x}\left(1-\frac{1}{2} F\right) .
\end{gathered}
$$

THEOREM 5.2. Suppose

(1) $a \in S, f$ and $h$ are functions from $S$ to $N$ such that $f(a)=$ $h(a)$ and $d h \in O B^{0}$, and

(2) $G, H, A$ and $B$ are functions from $S \times S$ to $N$ such that $(1-H)^{-1}$ exists and is $B, B$ is bounded, $B(d h) \in O A^{0}$, for $\{x, y\} \in S \times S$ $A(y, x)=[1-H(x, y)]^{-1}[1+G(x, y)]$ and $A-1 \in O B^{0}$ and

(3) $h$ is a constant function or ${ }_{x} \Pi^{y} A$ exists for each $\{x, y\} \in S \times S$. The following statements are equivalent:

(1) $H(x, y) f(y)+G(x, y) f(x) \in O A^{0}$ and

$$
f(x)=h(x)+(R L) \int_{a}^{x}(H f+G f)
$$

for each element $\{a, x\}$ of $S \times S$; and

(2) if $\{a, x, y\}$ is an 0-subdivision of $\{a, y\} \in S \times S$, then

$$
(L) \int_{a}^{y}|[\Pi A-A(,)] f()|=0
$$

and

$$
f(y)=\left({ }_{y} \Pi^{x} A\right) f(x)+(R) \int_{x}^{y}\left[\left({ }_{y} \Pi^{t} A\right) B(d h)\right] .
$$

Furthermore, if $f^{-1}$ exists and is bounded, then $(A-1) \in O M^{0}$.

\section{THEOREM 5.3. Suppose}

(1) $a \in S, f$ and $h$ are functions from $S$ to $N$ such that $f(a)=$ $h(a)$ and $d h \in O B^{0}$ and

(2) $G, H, A$ and $B$ are functions from $S \times S$ to $N$ such that $(1-H)^{-1}$ exists, $B(y, x)=[1-H(x, y)]^{-1},(1-H)^{-1} d h \in O A^{0},(B-1) \in O B^{0}$, $(B-1) \in O M^{0}, G \in O B^{0}, G \in O M^{0}$ and $A=1+G$. The following statements are equivalent: $S \times S$

(1) $H(x, y) f(y)+f(x) G(x, y) \in O A^{0}$ and for each element $\{a, x\} \in$

$$
f(x)=h(x)+(R L) \int_{a}^{x}(H f+f G) ; \quad \text { and }
$$

(2) if $\{a, x, y\}$ is an 0-subdivision of $\{a, y\} \in S \times S$, then

$$
\begin{aligned}
f(y)= & \left({ }_{y} \Pi^{x} B\right) f(x)\left({ }_{x} \Pi^{y} A\right) \\
& \left.+(R R) \int_{x}^{y}\left[{ }_{y} \Pi^{t} B\right)(1-H)^{-1}(d h)\left({ }_{t} \Pi^{y} A\right)\right] .
\end{aligned}
$$


THEOREM 5.4. Suppose

(1) $a \in S, f$ and $h$ are functions from $S$ to $N$ such that $f(a)=$ $h(a)$ and $d h \in O B^{0}$ and

(2) $G, H, A$ and $B$ are functions from $S \times S$ to $N$ such that $(1-H)^{-1}$ exists and is $B,(B-1) \in O B^{0},(B-1) \in O M^{0}, d h B \in O A^{0}$, $G \in O B^{0}, G \in O M^{0}$ and $A(y, x)=1+G(x, y) \in S \times S$. The following statements are equivalent: $S \times S$

(1) $f(y) H(x, y)+G(x, y) f(x) \in O A^{0}$ and for each element $\{a, x\} \in$

$$
f(x)=h(x)+(R L) \int_{a}^{x}(f H+G f) ; \quad \text { and }
$$

(2) if $\{a, x, y\}$ is an 0-subdivision of $\{a, y\} \in S \times S$, then

$$
\begin{aligned}
f(y)= & \left({ }_{y} \Pi^{x} A\right) f(x)\left({ }_{x} \Pi^{y} B\right) \\
& \left.+(R R) \int_{x}^{y}\left[{ }_{y} \Pi^{t} A\right)(d h) B\left({ }_{t} \Pi^{y} B\right)\right] .
\end{aligned}
$$

REMARK. The integrals $(R) \int_{a}^{x} f G,(L) \int_{a}^{x} f G,(R) \int_{a}^{x} G f,(L) \int_{a}^{x} G f,(M) \int_{a}^{x} f G$ and $(M) \int_{a}^{x} G f$ are special cases of the integrals $(R L) \int_{a}^{x}(f H+f G)$ and $(R L) \int_{a}^{x}(H f+G f)$ used in Theorems 5.1 and 5.2. Hildebrandt [4, p. $354]$ defines a modified Riemann-Stieltjes integral as follows $[g(x)$ has bounded variation and $g_{c}(x)$ denotes the continuous part of $g(x)$ ]: "If $\int_{a}^{b} f d g_{c}$ exists as an $R-S$ integral, then for any closed interval $(c, d)$ of $(a, b)$ we define

$$
\begin{aligned}
\int_{c}^{d} f(x) d g(x)= & \int_{c}^{d} f(x) d g_{c}(x)+f(c)[g(c+0)-g(c)] \\
& +\sum_{c<x<d} f(x)[g(x+0)-g(x-0)] \\
& +f(d)[g(d)-g(d-0)] . "
\end{aligned}
$$

This integral also simplifies to a special case of $(R L) \int_{c}^{d}(f H+f G)$ because the right member can be simplified as follows:

$$
\begin{aligned}
& R-S \int_{c}^{d} f(x) d g(x)= 1 / 2(R) \int_{c}^{d} f d g_{c} \\
&+1 / 2(L) \int_{c}^{d} f d g_{c} \\
&+\sum_{c<x \leqq d} f(x)[g(x)-g(x-0)] \\
&= 1 / 2(R) \int_{c}^{d} f d g_{c} f(x)[g(x+0)-g(x)] \\
&+1 / 2(L) \int_{c}^{d} f d g_{c}+(R) \int_{c}^{d} f d g_{l}+(L) \int_{c}^{d} f d g_{r}
\end{aligned}
$$




$$
\begin{aligned}
& =(R L) \int_{c}^{d}\left[f d\left(1 / 2 g_{c}+g_{l}\right)+f d\left(1 / 2 g_{c}+g_{r}\right)\right] \\
& =(R L) \int_{c}^{d}(f H+f G)
\end{aligned}
$$

where $g_{l}$ and $g_{r}$ are the functions of the "left breaks" and the "right breaks", respectively.

THEOREM 5.5. If $a \in S, f$ and $h$ are functions from $S$ to $N$ and $f^{-1}$ exists and is bounded, $H$ and $G$ are functions from $S \times S$ to $N$ such that $H$ and $f(x) G(x, y)$ are elements of $O A^{0}$ and, for each $\{a, x\} \in S \times S$, $\int_{a}^{x} H+h(x)=(L) \int_{a}^{x} f G$, then for $\{a, x\} \in S \times S$

$$
(L) \int_{a}^{x} f^{-1} H+(L) \int_{a}^{x} f^{-1} d h=\int_{a}^{x} G,
$$

provided two of the integrals exist.

Proof. Since $H$ and $f(x) G(x, y)$ are elements of $O A^{0}$, for each $x \in S$ and for each 0 -subdivision $\left\{x_{i}\right\}_{i=0}^{n}$ of $\{a, x\}$ there is a sequence $\left\{c_{i}\right\}_{i=1}^{n}$ of element of $N$ such that

$$
H\left(x_{i-1}, x_{i}\right)+h\left(x_{i}\right)-h\left(x_{i-1}\right)=f\left(x_{i-1}\right) G\left(x_{i-1}, x_{i}\right)+c_{i}
$$

and

$$
f^{-1}\left(x_{i-1}\right) H\left(x_{i-1}, x_{i}\right)+f^{-1}\left(x_{i-1}\right) d h_{i}=G\left(x_{i-1}, x_{i}\right)+f^{-1}\left(x_{i-1}, x_{i}\right) c_{i} .
$$

Since $f^{-1}$ is bounded and since $\sum_{i=1}^{n}\left|c_{i}\right|$ can be made arbitrarily small, it follows that for each $\{a, x\} \in S \times S$

$$
(L) \int_{a}^{x} f^{-1} H+(L) \int_{a}^{x} f^{-1} d h=\int_{a}^{x} G,
$$

provided two of the integrals exist.

THEOREM 5.6. Suppose $\{a, b\} \in S \times S, F$ and $G$ are functions from $S \times S$ to $N$ such that

$$
\int_{a}^{b}|F G|=0, \quad \int_{a}^{b}\left|G^{2}\right|=0,
$$

$F \in O B^{0}, G \in O B^{0},{ }_{a} \Pi^{b}(1+F)$ and ${ }_{a} \Pi^{b}(1+G)$ exist and $G(x, y), G(p, q)$, $F(x, y)$ and $F(p, q)$ commute for all elements $\{x, y\}$ and $\{p, q\}$ of $S \times S$; then

(1) ${ }_{a} \Pi^{b}(1+F)_{a} \Pi^{b}(1+G)={ }_{a} \Pi^{b}[1+(F+G)]$;

(2) $\left[\Pi_{a} \Pi^{b}(1+G)\right]^{n}={ }_{a} \Pi^{b}(1+n G)$, for $n$ a positive integer; and

(3) ${ }_{a} \Pi^{b}(1-G)=\left[\Pi_{a} \Pi^{b}(1+G)\right]^{-1}$, provided ${ }_{a} \Pi^{b}(1-G)$ exists. 
Proof of (1). If $\left\{x_{i}\right\}_{i=0}^{n}$ is an 0 -subdivision of $\{a, b\}$ then

$$
\begin{aligned}
& \left|\prod_{i=1}^{n}\left(1+F_{i}\right) \prod_{i=1}^{n}\left(1+G_{i}\right)-\prod_{i=1}^{n}\left[1+\left(F_{i}+G_{i}\right)\right]\right| \\
& \quad=\left|\prod_{i=1}^{n}\left(1+F_{i}+G_{i}+F_{i} G_{i}\right)-\prod_{i=1}^{n}\left[1+\left(F_{i}+G_{i}\right)\right]\right| \\
& \quad \leqq M^{2} \sum_{i=1}^{n}\left|F_{i} G_{i}\right|
\end{aligned}
$$

Since $\int_{a}^{b}|F G|=0$, it follows that

$$
{ }_{a} \Pi^{b}(1+F){ }_{a} \Pi^{b}(1+G)={ }_{a} \Pi^{b}[1+(F+G)] .
$$

Parts (2) and (3) are corollaries to part (1).

Theorems 5.7 and 5.8 are stated for matrices of complex numbers but these proofs can be extended to other rings, provided a function $g$ exists having properties similar to those of the function $g$ used in these proofs.

TheORem 5.7. If $A$ is a nonsingular $m \times m$ matrix of complex numbers and $n$ is a positive integer, then $A$ has $n$ distinct nth roots.

Proof. Since $A$ is nonsingular, there is only a finite number of values of $z$ for which $\operatorname{det}|1+z(A-1)|$ is zero; therefore, since $\operatorname{det}|1+z(A-1)|$ is not zero for a complex number $z$ near $(0,0)$ and $(1,0)$, there is a continuous function $g$ from the real numbers to the complex numbers such that $g(0)=(0,0), g(1)=(1,0), d g \in O B^{0}$ and $\operatorname{det}|1+g(x)(A-1)| \neq 0$. Let $M(x)=1+g(x)(A-1)$; then $M(0)=1$, $M(1)=A$, and $M^{-1}$ exists and is bounded on $\{0,1\}$. Let $H(x, y)=$ $M^{-1}(x)[M(y)-M(x)]$; then $M(x)=(L)_{0} \Pi^{x}\left(1+M^{-1} d M\right)=(L)_{0} \Pi^{x}(1+H)$, $H \in O M^{0}, H \in O B^{0}, \int_{0}^{1}\left|H^{2}\right|=0, H(p, q)$ and $H(r, t)$ commute for all number pairs $(p, q)$ and $(r, t)$. By Theorem 3.4, $H \in O A^{0}$; hence $(1 / n) H \in O A^{0}$, $(1 / n) H \in O M^{0}$ and $(L)_{0} \mathrm{II}^{x}[1+(1 / n) H]$ exists for $0 \leqq x \leqq 1$. For each $n$th root $a$ of the complex number $(1,0)$

$$
\begin{aligned}
\left\{a_{0} \Pi^{1}[1+(1 / n) H]\right\}^{n} & =a_{0}^{n} \Pi^{1}(1+H) \\
& =M(1)=A .
\end{aligned}
$$

Since $g$ is continuous, $\left\{_{0} \Pi^{1}[1+(1 / n) H]\right\}^{-1}$ exists; therefore each distinct $n$th root of the complex number $(1,0)$ gives a distinct $n$th root of $A$.

THEOREM 5.8. If $\left\{A_{i}\right\}_{i=1}^{\infty}$ is a sequence of $m \times m$ matrices of complex numbers such that $\sum_{i=1}^{\infty} A_{i}^{2}$ exists and, for $0<i \leqq j,\left|A_{i}\right|<1 / 4$ 
and $A_{i}$ and $A_{j}$ commute, then the following statements are equivalent:

(1) $\sum_{i=1}^{\infty} A_{i}$ exists and

(2) $\prod_{i=1}^{\infty}\left(1+A_{i}\right)$ exists.

Proof. Let $g$ be the function from the real numbers to the complex numbers such that $g(x)=(x, 0)$ and let $\left\{h_{i}\right\}_{i=1}^{\infty}$ be the sequence of functions such that for each positive integer $i, h_{i}(x)=1+A_{i} g(x)$. For each integer $i$ and each number $x, 0 \leqq x \leqq 1,\left|A_{i} g(x)\right|<1 / 2, h_{\imath}(0)=1, h_{i}(1)=$ $1+A_{i}, h_{i}^{-1}$ exists on $\{0,1\},\left|h_{i}^{-1}(x)\right|<2$ and

$$
\begin{aligned}
h_{i}(x) & =h_{i}(0) \cdot(L)_{0} \Pi^{x}\left(1+h_{i}^{-1} d h_{i}\right) \\
& =(L)_{0} \Pi^{x}\left[1+\left(1+A_{i} g\right)^{-1} A_{i} d g\right] \\
& =(L)_{0} \Pi^{x}\left\{1+\left[A_{i}-\left(1+A_{i} g\right)^{-1} A_{i}^{2} g\right] d g\right\} \\
& =(L)_{0} \Pi^{x}\left(1+A_{i} d g\right) \cdot(L)_{0} \Pi^{x}\left[1-\left(1+A_{i} g\right)^{-1} A_{i}^{2} g d g\right] .
\end{aligned}
$$

For each pair $m, n$ of positive integers, with $m<n$, there are elements $\alpha(m, n)$ and $\beta(m, n)$ of $N$ such that

$$
\begin{aligned}
\prod_{i=m}^{n}\left(1+A_{i}\right) & =\prod_{i=m}^{n} h_{i}(1) \\
& ={ }_{0} \Pi^{1}\left(1+\sum_{i=m}^{n} A_{i} d g\right){ }_{0} \Pi^{1}\left[1-\sum_{i=m}^{n}\left(1+A_{i} g\right)^{-1} A_{i}^{2} g d g\right] \\
& ={ }_{0} \Pi^{1}(1+B d g)[1+\alpha(m, n)],
\end{aligned}
$$

where

$$
\begin{aligned}
B & =\sum_{i=m}^{n} A_{i} \\
& ={ }_{0} \Pi^{1}(1+B d g)+\beta(m, n)
\end{aligned}
$$

and such that $\alpha(m, n) \rightarrow 0$ as $m, n \rightarrow \infty$ and, if ${ }_{0} \Pi^{1}(1+B d g)$ is uniformly bounded as $m, n \rightarrow \infty$, then $|\beta(m, n)| \rightarrow 0$ as $m, n \rightarrow \infty$.

$1 \rightarrow 2$. Since $\sum_{i=1}^{\infty} A_{i}$ exists, ${ }_{0} \Pi^{1}\left(1+\sum_{i=m}^{n} A_{i} d g\right)$ is uniformly bounded as $m, n \rightarrow \infty$ and $|\beta(m, n)| \rightarrow 0$ as $m, n \rightarrow \infty$. Let $m, n$ be an integer pair and $f$ be the function such that, for $0 \leqq x \leqq 1$, $f(x)=(L){ }_{0} \Pi^{x}(1+B d g)$, where $B=\sum_{i=m}^{n} A_{i}$; by Theorem 3.5 and 5.1, $f(x)=1+(L) \int_{0}^{x} f B d g$; hence,

$$
\begin{aligned}
\prod_{i=m}^{n}\left(1+A_{i}\right) & =f(1)+\beta(m, n) \\
& =1+(L) \int_{0}^{1} f B d g+\beta(m, n),
\end{aligned}
$$

and 


$$
\begin{aligned}
\left|\prod_{i=m}^{n}\left(1+A_{i}\right)-1\right| & =\left|\int_{0}^{1} f B d g+\beta(m, n)\right| \\
& \leqq|B| \cdot|f|_{\max } \cdot \sum_{\max }|d g|+|\beta(m, n)| .
\end{aligned}
$$

Since there are maximum values for $f(x)$ and $\Sigma|d g|$ on $\{0,1\}$ which are independent of $m$ and $n$ and since $|\beta(m, n)|$ and $\left|\sum_{i=m}^{n} A_{i}\right|=|B|$ can be made arbitrarily small, it follows that $\left|\prod_{i=m}^{n}(1+A)-1\right| \rightarrow 0$ as $m, n \rightarrow \infty$. Since the sequence $\left\{\prod_{i=1}^{n}\left(1+A_{i}\right)\right\}_{n=1}^{\infty}$ is bounded,

$$
\lim _{n \rightarrow \infty} \prod_{i=1}^{n}\left(1+A_{i}\right)
$$

exists. Since for each integer $i$

$$
h_{i}^{-1}(x)=h_{i}^{-1}(0) \cdot(L)_{0} \Pi^{x}\left(1-h_{i}^{-1} d h_{i}\right),
$$

by repeating the above manipulations for $h_{i}^{-1}$ and for $\prod_{i=m}^{n}\left(1+A_{i}\right)^{-1}$, we can show that $\lim _{n \rightarrow \infty} \prod_{i=1}^{n}\left(1+A_{i}\right)^{-1}$ exists and then show that

$$
\lim _{n \rightarrow \infty} \prod_{i=1}^{n}\left(1+A_{i}\right)^{-1}=\left[\lim _{n \rightarrow \infty} \prod_{i=1}^{n}\left(1+A_{i}\right)\right]^{-1} \text {. }
$$

$2 \rightarrow 1$. Since $d g \in O B^{0},{ }_{0} \Pi^{1}\left(1+\sum_{i=m}^{n} A_{i} d g\right)$ exists for each pair of positive integers $m, n$. Since $\prod_{i=1}^{\infty}\left(1+A_{i}\right)$ and $\left[\prod_{i=1}^{\infty}\left(1+A_{i}\right)\right]^{-1}$ exists and since $|\alpha(m, n)| \rightarrow 0$ as $m, n \rightarrow \infty$, it follows that $\left\{\prod_{i=1}^{n}\left(1+A_{i}\right)\right\}_{n=1}^{\infty}$ and $\left\{\prod_{i=1}^{n}\left(1+A_{i}\right)^{-1}\right\}_{n=1}^{\infty}$ are bounded and $|\beta(m, n)| \rightarrow 0$ as $m, n \rightarrow \infty$.

If $2 \nrightarrow 1$, there is a positive number $c$ such that if $J$ is an integer there are integers $n \geqq m>J$ such that $1 / 4>\left|\sum_{i=m}^{n} A_{i}\right|>c$. Therefore, there are positive integers $m$ and $n$ such that $c / 6>\left|\prod_{i=m}^{n}\left(1+A_{i}\right)-1\right|$, $c / 6>|\beta(m, n)|$ and $1 / 4>\left|\sum_{i=m}^{n} A_{i}\right|>c$. Since ${ }_{0} \Pi^{1}\left(1+\sum_{i=m}^{n} A_{i} d g\right)$ exists, there is an 0 -subdivision $\left\{x_{j}\right\}_{j=0}^{p}$ of $\{0,1\}$ of evenly spaced numbers such that if $v=g\left(x_{1}\right)-g\left(x_{0}\right), B=\sum_{i=m}^{n} A_{i}$ and

$$
u={ }_{0} \Pi^{1}(1+B d g)-\prod_{j=1}^{p}\left\{1+B\left[g\left(x_{j}\right)-g\left(x_{j-1}\right)\right]\right\},
$$

then

$$
u={ }_{0} \Pi^{1}(1+B d g)-(1+B v)^{p}
$$

and $c / 6>|u|$. Hence,

$$
\begin{aligned}
c / 6 & >\left|\prod_{i=m}^{n}\left(1+A_{i}\right)-1\right| \\
& =\left|\Pi_{0} \Pi^{1}(1+B d g)+\beta(m, n)-1\right| \\
& \geqq\left|(1+B v)^{p}-1\right|-|\beta(m, n)|-|u| .
\end{aligned}
$$

By using the binomial theorem and the above inequalities, we obtain 


$$
\begin{aligned}
c / 2> & \left|(1+B v)^{p}-1\right| \\
= & \mid 1+p B v+p(p-1)(B v)^{2} / 2 ! \\
& +p(p-1)(p-2)(B v)^{3} / 3 !+\cdots+(B v)^{p}-1 \mid \\
= & |B|-\left[|B|^{2}|p v||(p-1) v| / 2 !\right. \\
& \left.+|B|^{3}|p v||(p-1) v||(p-2) v| / 3 !+\cdots+|B|^{p}|v|^{p}\right] .
\end{aligned}
$$

If $0 \leqq j \leqq p,|(p-j) v| \leqq 1$; therefore,

$$
c / 2>|B|-|B|\left[\frac{1}{4} \cdot \frac{1}{2 !}+\left(\frac{1}{4}\right)^{2} \cdot \frac{1}{3 !}+\cdots\right]>\frac{1}{2}|B| .
$$

Hence, $c>|B|=\left|\sum_{\imath=m}^{n} A_{i}\right|>c$; this contradiction proves that $2 \rightarrow 1$.

6. Solutions for integral equations. In this section we present several examples to show how product integrals can be used in the solution of several types of integral equations. In each example we assume that all elements commute, that all the preceding theorems hold when needed and that functions such as $n f, f^{\dot{n}}$, and $f^{-1}$ exist for $n$ a rational number. Lower case letters will be used for functions from $S$ to $N$ and capitals for functions from $S \times S$ to $N$. All integrals will be of the type $(L) \int_{a}^{b} f d g$ and $(L)_{a} \Pi^{b}(1+f d g)$. Observe that

$$
f(x)=f(a)(L)_{a} \Pi^{x}\left(1+f^{-1} d f\right)
$$

and that, under the conditions stated above,

$$
\begin{aligned}
{[f(x)]^{n} } & =[f(a)]^{n}(L)_{a} \Pi^{x}\left(1+n f^{-1} d f\right) \\
& =[f(a)]^{n}+(L) \int_{a}^{x} n f^{n-1} d f .
\end{aligned}
$$

EXAMPLe 1. $(n+1) \int_{a}^{b} f^{n} d f=[f(b)]^{n+1}-[f(a)]^{n+1}$.

$$
\text { Proof. } \quad \begin{aligned}
{[f(x)]^{n+1} } & =[f(a)]^{n+1}{ }_{a} \Pi^{x}\left[1+(n+1) f^{-1} d f\right] \\
& =[f(a)]^{n+1}+(n+1) \int_{a}^{x} f^{n} d f .
\end{aligned}
$$

EXAMPLE 2. $\int_{a}^{b} g^{-2}(g d f-f d g)=f(b) g^{-1}(b)-f(a) g^{-1}(a)$.

$$
\text { Proof. } \begin{aligned}
f(x) g^{-1}(x) & =f(a)_{a} \Pi^{x}\left(1+f^{-1} d f\right) g^{-1}(a)_{a} \Pi^{x}\left(1-g^{-1} d g\right) \\
& =f(a) g^{-1}(a)_{a} \Pi^{x}\left(1+f^{-1} d f-g^{-1} d g\right) \\
& =f(a) g^{-1}(a)+\int_{a}^{x} f g^{-1}\left(f^{-1} d f-g^{-1} d g\right) .
\end{aligned}
$$


EXAMPLE 3. $\int_{a}^{b}(f h d g+f d h g+d f h g)=f(b) h(b) g(b)-f(a) h(a) g(a)$.

Proof. $f(x) h(x) g(x)=f(a) h(\alpha) g(a)_{a} \Pi^{x}\left(1+f^{-1} d f+g^{-1} d g+h^{-1} d h\right)$ $=f(a) h(a) g(a)+\int_{a}^{x} f h g\left(f^{-1} d f+g^{-1} d g+h^{-1} d h\right)$.

EXAMPLE 4. If $y(x)=y(a)+\int_{a}^{x}\left(y G+y^{n} F\right)$ and $n \neq 1$, then $y(x)={ }_{a} \Pi^{x}(1+G)\left\{[y(a)]^{1-n}+(1-n) \int_{a}^{x}[\Pi(1-G)]^{1-n} F\right\}^{1 /(1-n)}$.

Proof. $y(x)=y(a)_{a} \Pi^{x}\left(1+G+y^{n-1} F\right)$

$$
=y(a)_{a} \Pi^{x}\left(1+y^{n-1} F\right)_{a} \Pi^{x}(1+G)
$$$$
\left[y(x)_{a} \Pi^{x}(1-G)\right]^{1-n}=[y(a)]^{1-n} \Pi^{x}\left[1+(1-n) y^{n-1} F\right]
$$$$
=[y(a)]^{1-n}+(1-n) \int_{a}^{x}[\Pi(1-G)]^{1-n} F .
$$

EXAMPle 5. If $k$ is a constant and

$$
y(x)=y(a)+\int_{a}^{x}(y+k)(h y+f) G,
$$

then

$$
\begin{aligned}
y(x)+k= & { }_{a} \Pi^{x}[1+(f-h k) G] \\
& \times\left\{[y(a)+k]^{-1}-\int_{a}^{x} \Pi^{t}[1+(f-h k) G] h G\right\}^{-1} .
\end{aligned}
$$

Proof.

$$
\begin{aligned}
& y(x)+k=[y(a)+k]_{a} \Pi^{x}[1+(h y+f) G] \\
& =[y(a)+k]_{a} \Pi^{x}[1+(h y+h k) G]_{a} \Pi^{x}[1+(f-h k) G] \\
& \left\{[y(x)+k]_{a} \Pi^{x}[1-(f-h k) G]\right\}^{-1} \\
& \quad=[y(a)+k]^{-1}{ }_{a} \Pi^{x}[1-(y+k) h G] \\
& \quad=[y(a)+k]^{-1}-\int_{a}^{x} \Pi^{t}[1+(f-h k) G] h G .
\end{aligned}
$$

EXAMPLE 6. If $f(x) u(x)=f(a)+\int_{a}^{x} f^{p} G$, then $f(x)=$ ? Indication of proof. $f(x) u(x)=f(a)+\int_{a}^{x}\left[f^{p} u^{p}\right] u^{-p} G$; the remainder of this proof is similar to Example 4.

EXAMPLE 7. If $y(x)+(p+q) \int_{a}^{x} y G+p q \int_{a}^{x}\left(\int_{a}^{t} y G\right) G=f(x)$, where $p$ and $q$ are constants, $y(x)=$ ? 
Indication of proof. The given equation can be rewritten

$$
\left[y(x)+p \int_{a}^{x} y G\right]+\int_{a}^{x}\left[y(t)+p \int_{a}^{t} y G\right] q G=f(x),
$$

which is covered by Theorem 5.1 with $y(t)+p \int_{a}^{t} y G$ treated as one function. Then Theorem 5.1 is used a second time.

EXAMPLE 8. If $y(x)=c+\int_{a}^{x}(y+p)^{1 / 2}(y+q)^{1 / 2} G$, and $c, p$ and $q$ are constants, $y(x)=$ ?

Indication of proof.

$$
\begin{aligned}
{[y(x)+p]^{1 / 2} } & =(c+p)_{a}^{1 / 2} \Pi^{x}\left[1+\frac{1}{2}(y+p)^{-1 / 2}(y+q)^{1 / 2} G\right] \\
& =(c+p)^{1 / 2}+\frac{1}{2} \int_{a}^{x}(y+q)^{1 / 2} G .
\end{aligned}
$$

Similarly,

$$
[y(x)+q]^{1 / 2}=(c+q)^{1 / 2}+\frac{1}{2} \int_{a}^{x}(y+p)^{1 / 2} G .
$$

Substituting $[y(t)+q]^{1 / 2}$ in the preceding equation gives

$$
[y(x)+p]^{1 / 2}=(c+p)^{1 / 2}+\frac{1}{2} \int_{a}^{x}\left[(c+q)^{1 / 2}+\frac{1}{2} \int_{a}^{t}(y+p)^{1 / 2} G\right] G,
$$

and this can be rewritten as a special case of Example 7,

$$
\begin{aligned}
\left\{[y(x)+p]^{1 / 2}\right. & \left.-\frac{1}{2} \int_{a}^{x}(y+p)^{1 / 2} G\right\} \\
& +\frac{1}{2} \int_{a}^{x}\left[(y+p)^{1 / 2}-\frac{1}{2} \int_{a}^{t}(y+p)^{1 / 2} G\right] G \\
= & (c+p)^{1 / 2}+\frac{1}{2} \int_{a}^{x}(c+q)^{1 / 2} G .
\end{aligned}
$$

ExAmPLe 9. If $k$ is a constant and $G, s$ and $c$ are functions such that $s(x)=k+\int_{a}^{x} c G$ and $c(x)=-\int_{a}^{x} s G$, then $s^{2}(x)+c^{2}(x)=k^{2}$.

Proof. Since $s(x)=k_{a} \Pi^{x}\left(1+s^{-1} c G\right)$, then

$$
\begin{aligned}
s^{2}(x) & =k^{2}{ }_{a} \Pi^{x}\left(1+2 s^{-1} c G\right)=k^{2}+2 \int_{a}^{x} s c G \\
& =k^{2}-2 \int_{a}^{x}\left(\int_{a}^{t} s G\right) s G=k^{2}-\left(\int_{a}^{x} s G\right)^{2}=k^{2}-c^{2}(x) .
\end{aligned}
$$


REMARK. In the preceding examples the function $G(x, y)$ was used instead of the function $d g$ because $G(x, y)$ might be a more general function such as

$$
G(x, y)=f(x) h(y)[g(y)-g(x)][p(x)+q(y)] .
$$

\section{BIBLIOGRAPHY}

1. W. D. L. Appling, Concerning nonnegative valued interval functions, Proc. Amer. Math. Soc. 13, No. 5 (1962), 784-788.

2. Infinite series and nonnegative valued interval functions, Duke Math. J. 30, No. 1 (1963), 107-112.

3. T. H. Hildebrandt, Definitions of Stieltjes integrals of the Riemann type, Amer. Math. Monthly, 45 (1938), 265-278.

4. On systems of linear differentio-Stieltjes-integral equations, Illinois J. Math. 3 (1959), 352-373.

5. J. S. MacNerney, Continuous products in linear spaces, J. Elisha Mitchell Sci. Soc. 71 (1955), 185-200.

6. Concerning quasi-harmonic operators, J. Elisha Mitchell Sci. Soc. 73 (1957), 257-261.

7. - Integral equations and semigroups, Illinois J. Math. 7 (1963), 148-173.

8. P. R. Masani, Multiplicative Riemann integration in normed rings, Trans. Amer. Math. Soc. 61 (1947), 147-192.

9. H. S. Wall, Concerning harmonic matrices, Arch. Math., 5 (1954), 160-167.

Southwest Texas State College 


\section{PACIFIC JOURNAL OF MATHEMATICS}

\section{EDITORS}

\section{H. SAMELSON}

Stanford University

Stanford, California

\section{R. M. BLUMenthaL \\ University of Washington \\ Seattle, Washington 98105}

*J. DugundjI

University of Southern California Los Angeles, California 90007

\section{RICHARD ARENS}

University of California

Los Angeles, California 90024

\section{ASSOCIATE EDITORS}
E. F. BECKENBACH
B. H. NeUManN
F. WOLF
K. YosIDA

\section{SUPPORTING INSTITUTIONS}

\author{
UNIVERSITY OF BRITISH COLUMBIA \\ CALIFORNIA INSTITUTE OF TECHNOLOGY \\ UNIVERSITY OF CALIFORNIA \\ MONTANA STATE UNIVERSITY \\ UNIVERSITY OF NEVADA \\ NEW MEXICO STATE UNIVERSITY \\ OREGON STATE UNIVERSITY \\ UNIVERSITY OF OREGON \\ OSAKA UNIVERSITY \\ UNIVERSITY OF SOUTHERN CALIFORNIA
}

\author{
STANFORD UNIVERSITY \\ UNIVERSITY OF TOKYO \\ UNIVERSITY OF UTAH \\ WASHINGTON STATE UNIVERSITY \\ UNIVERSITY OF WASHINGTON \\ AMERICAN MATHEMATICAL SOCIETY \\ CHEVRON RESEARCH CORPORATION \\ TRW SYSTEMS \\ NAVAL ORDNANCE TEST STATION
}

Mathematical papers intended for publication in the Pacific Journal of Mathematics should be typewritten (double spaced). The first paragraph or two must be capable of being used separately as a synopsis of the entire paper. It should not contain references to the bibliography. Manuscripts may be sent to any one of the four editors. All other communications to the editors should be addressed to the managing editor, Richard Arens at the University of California, Los Angeles, California 90024.

50 reprints per author of each article are furnished free of charge; additional copies may be obtained at cost in multiples of 50 .

The Pacific Journal of Mathematics is published monthly. Effective with Volume 16 the price per volume (3 numbers) is $\$ 8.00$; single issues, $\$ 3.00$. Special price for current issues to individual faculty members of supporting institutions and to individual members of the American Mathematical Society: $\$ 4.00$ per volume; single issues $\$ 1.50$. Back numbers are available.

Subscriptions, orders for back numbers, and changes of address should be sent to Pacific Journal of Mathematics, 103 Highland Boulevard, Berkeley 8, California.

Printed at Kokusai Bunken Insatsusha (International Academic Printing Co., Ltd.), No. 6, 2-chome, Fujimi-cho, Chiyoda-ku, Tokyo, Japan.

PUBLISHED BY PACIFIC JOURNAL OF MATHEMATICS, A NON-PROFIT CORPORATION

The Supporting Institutions listed above contribute to the cost of publication of this Journal, but they are not owners or publishers and have no responsibility for its content or policies.

* Paul A. White, Acting Editor until J. Dugundji returns. 


\section{Pacific Journal of Mathematics}

\section{Vol. 16, No. 2 December, 1966}

Loren N. Argabright, Invariant means on topological semigroups ........ 193

William Arveson, A theorem on the action of abelian unitary groups ...... 205

John Spurgeon Bradley, Adjoint quasi-differential operators of Euler

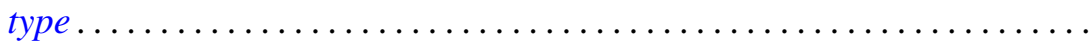

Don Deckard and Lincoln Kearney Durst, Unique factorization in power series rings and semigroups ........................... 239

Allen Devinatz, The deficiency index of ordinary self-adjoint differential operators..................................... 243

Robert E. Edwards, Operators commuting with translations ............ 259

Avner Friedman, Differentiability of solutions of ordinary differential equations in Hilbert space .............................. 267

Boris Garfinkel and Gregory Thomas McAllister, Jr., Singularities in a variational problem with an inequality ......................

Seymour Ginsburg and Edwin Spanier, Semigroups, Presburger formulas,

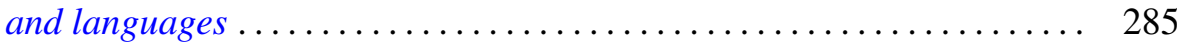

Burrell Washington Helton, Integral equations and product integrals . . . . . . 297

Edgar J. Howard, First and second category Abelian groups with the n-adic topology.........................................

Arthur H. Kruse and Paul William Liebnitz, Jr., An application of a family homotopy extension theorem to ANR spaces.

Albert Marden, I. Richards and Burton Rodin, On the regions bounded by homotopic curves

Willard Miller, Jr., A branching law for the symplectic groups ...

Marc Aristide Rieffel, A characterization of the group algebras of the finite

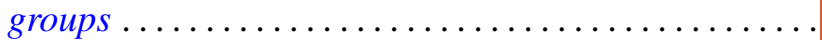

P. P. Saworotnow, On two-sided $H^{*}$-algebras

John Griggs Thompson, Factorizations of p-solvable groups ...

Shih-hsiung Tung, Harnack's inequalities on the classical Cartan

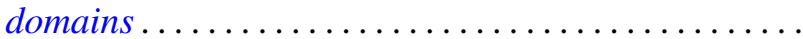

